\title{
Monoamine neurotransmitters modulate NT-3 levels in astrocytes
} Damijana M Jurič

\author{
Address: Department of Pharmacology and Experimental Toxicology, Faculty of Medicine, University of Ljubljana, 1000 Ljubljana, Slovenia \\ Email: Damijana M Jurič - damijana-mojca.juric@mf.uni-lj.si
}

from I5th Scientific Symposium of the Austrian Pharmacological Society (APHAR) Joint meeting with the Hungarian Society of Experimental and Clinical Pharmacology (MFT) and the Slovenian Pharmacological Society (SDF)

Graz, Austria. 19-21 November 2009

Published: 12 November 2009

BMC Pharmacology 2009, 9(Suppl 2):A60 doi:10.1 I86/I47I-22I0-9-S2-A60

This abstract is available from: http://www.biomedcentral.com/I47I-22 I0/9/S2/A60

(c) 2009 Jurič; licensee BioMed Central Ltd.

\section{Background}

Neurotrophin-3 (NT-3), a member of the neurotrophin family of neurotrophic factors, displays profound neuromodulatory functions in the normal and in the diseased brain. Under physiological conditions, NT-3 is produced by neuronal cells and also by local glial cells. We focused our investigation on the ability of astrocytes to synthesize NT-3 and, additionally, on the active involvement of the neurotransmitters noradrenaline, adrenaline, dopamine, histamine and serotonin (5-HT) in the regulation of NT-3 production in neonatal rat cortical astrocytes.

\section{Results}

Our study confirms the ability of neonatal rat cortical and cerebellar astrocytes in primary culture to express and synthesize significant amounts of NT-3. The examined monoamines, with the exception of 5-HT are able to potently and transiently increase NT-3 mRNA and NT-3 protein cell levels; their action is dose- and time-dependent. Screening different activators of basic intracellular second messenger systems which can participate in the possible monoamine receptor mediated stimulation of NT-3 by examined monoamines revealed that forskolin, dibutyryl cAMP (dBcAMP), as well as calcimycin $\left(\mathrm{Ca}^{2+}\right.$ ionophore A23187) and phorbol 12-myristate 13-acetate (TPA), markedly increase the cellular level of NT-3 protein. Neurotransmitter-induced NT-3 is susceptible (to varying degrees) to inhibition by $\mathrm{H}-89$ (an inhibitor of protein kinase A, PKA) or staurosporin (an inhibitor of protein kinase $\mathrm{C}, \mathrm{PKC}$ ), which led us to conclude that downstream signaling responsible for the stimulation of NT-3 synthesis by monoamines in astrocytes is a receptor- mediated process consisting of multiple, complex intracellular mechanisms involving the CAMP/PKA pathway, activation of PKC, as well as mobilization of $\mathrm{Ca}^{2+}$ ions.

\section{Conclusion}

In conclusion, our study indicates for the first time that monoaminergic neurotransmitters play an important role in the regulation of neurotropic NT-3 activity in cultured rat astrocytes. 